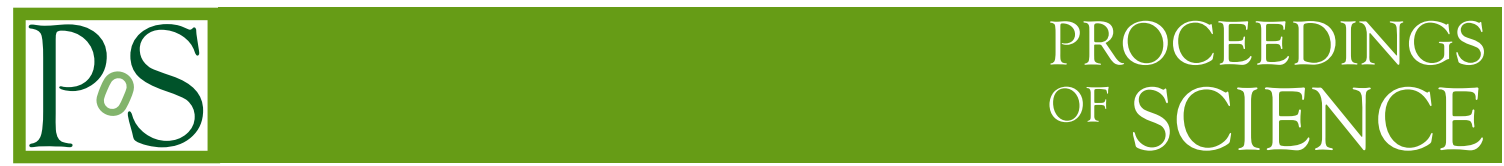

\title{
CMB probes on generally correlated axion isocurvature perturbations
}

\section{Kenji Kadota*}

Center for Theoretical Physics of the Universe, Institute for Basic Science,

Daejeon, 34051, Korea

E-mail: kadota@ibs.re.kr

\begin{abstract}
We discuss the future prospects for measuring the isocurvature perturbations which are correlated with the curvature perturbations. We show how precisely the forthcoming CMB experiments can constrain such correlated isocurvature perturbations, and, as concrete examples, illustrate how the correlated isocurvature perturbations help in discriminating the axion inflation scenarios.
\end{abstract}

The 11th International Workshop Dark Side of the Universe 2015

14-18 December 2015

Kyoto, Japan

*Speaker. 


\section{Introduction}

With the precise measurements of the CMB observables, we can now robustly constrain the cosmological parameters even in the existence of the cross-correlated isocurvature modes. The robustness here refers to the fact that the parameter estimations do not significantly change the bestfit conventional $\Lambda \mathrm{CDM}$ parameters even after including the cross-correlations which can induce the degeneracies among cosmological parameters. The WMAP for instance suffered from the big degeneracies in including the isocurvature perturbations, and we needed to wait for the Planck to robustly constrain the cosmological parameters when the isocurvature perturbations have the nontrivial cross correlations with the curvature perturbations. It would hence be worth exploring now the inflation scenarios where such cross-correlated isocurvature perturbations arise. We first show how precisely the forthcoming CMB experiments can potentially constrain the cross-correlated isocurvature modes [1]. We then present how the cross-correlated isocurvature perturbations can arise in the axion inflation scenarios as concrete examples, where we also illustrate how the crosscorrelations can help in distinguishing different inflation scenarios [2].

\section{CMB probes on correlated isocurvature perturbations}

We first show how much the cosmological parameter estimations can be affected by the crosscorrelated isocurvature perturbations. We performed the Fisher matrix analysis for forecasting the bounds on the cosmological parameters assuming a Planck-like CMB satellite experiment. The CMB observables $(T, E, L)$ of our interest are, respectively, the CMB temperature, $E$-mode polarization and the $\mathrm{CMB}$ deflection angle representing the CMB lensing [3].

For our parameter estimations, we define the power spectra of the curvature, isocurvature and their cross-correlation, denoted by subscripts $R, I$ and $C$ respectively, as

$$
P_{X}=A_{X}\left(k_{0}\right)\left(\frac{k}{k_{0}}\right)^{n_{X}-1}
$$

with $X \supset(R, I, C)$, and the fractions of the isocurvature perturbation and cross-correlation are defined as

$$
\beta_{I}=\frac{P_{I}}{P_{R}}, \beta_{C}=\frac{P_{C}}{\sqrt{P_{R} P_{I}}}
$$

The total power spectrum which we observe is the sum of $P_{R}, P_{I}$ and $P_{C}$. Unless stated otherwise, $A$ 's and $\beta$ 's are evaluated at the reference scale $k_{0}=0.05 \mathrm{Mpc}^{-1}$ and the isocurvature fraction is set to $\beta_{I}=0.04$ (which corresponds to 95\% CL upper bound from Planck+WMAP [4] when there is no correlation between the curvature and isocurvature perturbations) in the following analysis.

The Fisher matrix consists of 9 parameters, $\left(A_{I}, A_{C}, n_{I}\right)$ in addition to the conventional six $\Lambda \mathrm{CDM}$ parameters $\left(\Omega_{\Lambda}=0.69, \Omega_{m} h^{2}=0.14, \Omega_{b} h^{2}=0.022, n_{R}=0.96, A_{R}=2.2 \times 10^{-9}, \tau\right.$ (reionization optical depth $=0.095$ ) with the numerical values being the fiducial values in our Fisher analysis [5]. The spectral index of the cross-correlation is set to $n_{C}=\left(n_{R}+n_{I}\right) / 2$ for simplicity, which is indeed realized in and motivated from our axion scenarios to be discussed in the next section. The marginalized errors for the parameters involving the isocurvature perturbation are listed in Table 1 for different cross-correlation power spectrum amplitudes. We find that $\beta_{C} \leq O(0.1)$ is 


\begin{tabular}{|c||c|c|c|c|}
\hline & $T$ & $T E$ & $T L$ & Joint \\
\hline \hline$\beta_{C}=1$ & & & & \\
$\sigma\left(A_{C}\right) / A_{C}$ & 52 & 3.8 & 7.6 & 3.8 \\
$\sigma\left(A_{I}\right) / A_{I}$ & 52 & 12 & 30 & 12 \\
$\sigma\left(n_{I}\right) / n_{I}$ & 11 & 4.3 & 6.7 & 4.2 \\
\hline$\beta_{C}=0.1$ & & & & \\
$\sigma\left(A_{C}\right) / A_{C}$ & 98 & 24 & 55 & 23 \\
$\sigma\left(A_{I}\right) / A_{I}$ & 67 & 13 & 31 & 13 \\
$\sigma\left(n_{I}\right) / n_{I}$ & 17 & 6.1 & 9.9 & 6.0 \\
\hline$\beta_{C}=0.01$ & & & & \\
$\sigma\left(A_{C}\right) / A_{C}$ & 600 & 230 & 540 & 220 \\
$\sigma\left(A_{I}\right) / A_{I}$ & 70 & 13 & 32 & 13 \\
$\sigma\left(n_{I}\right) / n_{I}$ & 18 & 6.4 & 11 & 6.3 \\
\hline
\end{tabular}

Table 1: $1 \sigma$ errors [\%] for different values of $\beta_{C} . T$ refers to the analysis using only the CMB temperature data. $T E(T L)$ refers to the analysis using both temperature and polarization (temperature and lensing) information. Joint refers to the use of $T, E$ and $L$.

required for the error on $A_{C}$ not to exceed $100 \%$. We here point out the importance of the CMB polarization data in the parameter estimation for its breaking the degeneracy among the cosmological parameters. It, in particular, breaks the degeneracy between $\tau$ and $A_{C}$ which arises because the power spectrum amplitude is suppressed by the reionization optical depth by a factor $\sim e^{-2 \tau}$. This is illustrated in Figure 1. We can clearly see the big degeneracy between $\tau$ and $A_{C}$ in the temperature data alone, which is broken by adding the polarization data. Polarization is sensitive to the reionization bump on large scales $(\ell \lesssim 10)$ which can lift the degeneracies concerning $\tau$, resulting in the improved constraints on $A_{C}$.

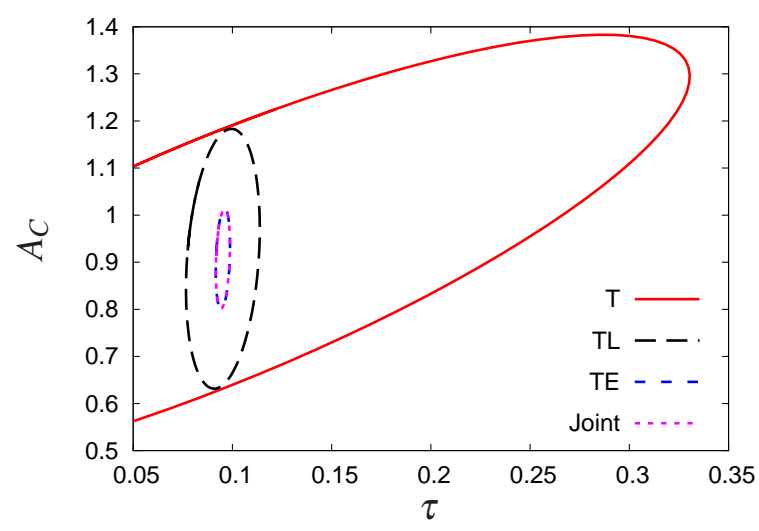

Figure 1: Marginalized $1 \sigma$ error contours for $\tau$ and $A_{C}$ (for $\beta_{I}=0.04, \beta_{C}=1$ ).

The cosmological parameters are in fact not totally independent from each other, and the existence of a small cross-correlation power spectrum can well affect the other cosmological parameters which are tightly constrained by the CMB alone. This is illustrated in Table 2 where the errors are 


\begin{tabular}{|c||c|c|c|c|c|c|}
\hline & $\Omega_{\Lambda}$ & $\Omega_{m} h^{2}$ & $\Omega_{b} h^{2}$ & $n_{R}$ & $A_{R}$ & $\tau$ \\
\hline \hline$\beta_{C}=1$ & 1.1 & 1.1 & 1.0 & 1.4 & 0.82 & 0.79 \\
$\beta_{C}=0.01$ & 1.1 & 1.1 & 1.0 & 1.4 & 0.95 & 0.93 \\
No correlation & 1.0 & 1.0 & 1.0 & 1.1 & 0.95 & 0.93 \\
\hline
\end{tabular}

Table 2: Normalized error $\sigma / \sigma_{\text {no iso. }}$ The comparison between the error estimation assuming the isocurvature perturbation $\left(\beta_{I}=0.04\right)$ and that assuming the $\Lambda \mathrm{CDM}$ with no isocurvature perturbation. All the errors here are estimated combining all of $T, E$ and $L$.

normalized to those assuming no isocurvature components. The marginalized errors in this table are calculated by using the $9 \times 9$ Fisher matrix except the last row with no cross-correlation which uses $8 \times 8$ Fisher matrix without $A_{C}$. These errors are then divided by those calculated by $6 \times 6$ Fisher matrix in the $\Lambda \mathrm{CDM}$ model. The error in $\tau$ is reduced partly because the response of polarization to the isocurvature perturbations is different from that to the adiabatic perturbations. This as a result also helps in reducing the errors in $A_{R}$ by breaking the $\tau$ - $A_{R}$ degeneracy. We can see that the estimation of some of the $\Lambda \mathrm{CDM}$ parameters can well be affected by $O(10) \%$ in existence of the cross-correlation, and the complete ignorance of the cross-correlation could result in the misinterpretation of the underlying cosmological model.

\section{Axion inflation scenarios with cross-correlated isocurvature perturbations}

We now discuss concrete inflation models where the cross-correlated isocurvature perturbations can arise. We discuss here the natural inflation [6] as an example where the inflation is induced by an axion inflaton field while there exists another axion whose energy density during inflation is sub-dominant and hence induces the isocurvature perturbations. The cross-correlation between the curvature and isocurvature perturbations can arise from the interaction term

$$
V_{\text {int }}=\Lambda_{2}^{4}\left(1-\cos \left(\frac{\phi}{g_{1}}+\frac{\chi}{g_{2}}\right)\right),
$$

where $\phi$ and $\chi$ represent, respectively, an axion-inflaton and another light axion field. The inflation is induced from an axion-inflaton potential

$$
V_{\mathrm{inf}}=\Lambda_{1}^{4}\left(1-\cos \frac{\phi}{f}\right)
$$

with $f$ being the axion decay constant.

For the notational brevity, we in the following define the parameters

$$
\sigma=\frac{\phi}{f}, \psi=\frac{\phi}{g_{1}}, \theta=\frac{\chi}{g_{2}},
$$

so that the total potential can be written as

$$
V=\Lambda_{1}^{4}(1-\cos \sigma)+\Lambda_{2}^{4}(1-\cos (\psi+\theta)) .
$$

We hereafter focus on the scenarios where the adiabatic perturbations are dominantly sourced by the axion-inflaton fluctuation $\delta \phi$ and the additional axion fluctuation $\delta \chi$ leads to the isocurvature 
perturbations. The cross-correlation between the curvature and isocurvature perturbations can be obtained, using the in-in formalism [1,7],

$$
P_{C}=4.2\left(\frac{1}{g_{1} g_{2}}\right)\left(\frac{\Lambda_{2}}{\Lambda_{1}}\right)^{4}\left(\frac{f}{\phi_{0}}\right)^{2} \cos \left(\psi_{0}+\theta_{0}\right) \sqrt{P_{R} P_{I}},
$$

and Fig. 2 shows the following cross-correlation parameter

$$
\beta_{C} \equiv \frac{P_{C}}{\sqrt{P_{R} P_{I}}} \sim 4.2\left(\frac{1}{g_{1} g_{2}}\right)\left(\frac{\Lambda_{2}}{\Lambda_{1}}\right)^{4}\left(\frac{f}{\phi_{0}}\right)^{2} \cos \left(\psi_{0}+\theta_{0}\right)
$$

The subscript 0 represents the background field values during the inflation. In the figure, we vary the axion decay constant $f$ from 1 to 20 and the e-folding number $N$ from 50 to 60 . Here, the prefactor of $\phi_{0}^{2}$ is set to be of order 0.001 and the power spectrum of the adiabatic curvature perturbations

$$
P_{R}=A_{S} k^{n_{s}-1},
$$

is fixed to be $A_{S} \simeq H^{2} /\left(8 \pi^{2} \varepsilon\right) \simeq 2.2 \times 10^{-9}$ with $\varepsilon \simeq 2 / \phi_{0}^{2}$ being the slow-roll parameter at the pivot scale $k_{*}=0.05 \mathrm{Mpc}^{-1}$. The high- $l(l \geq 30) T E, E E$ data turn out to drive the isocurvature cross-correlation towards a smaller value and disfavor the negative cross-correlations which would be allowed otherwise with the high-l TT data [8]. We can find that the coefficient $c$ in $\beta_{C}=c \phi_{0}^{2}$ has to be of order less than $10^{-3}$ to be within 2 sigma and the axion decay constant $f$ is constrained to the range between 5 and 10 .

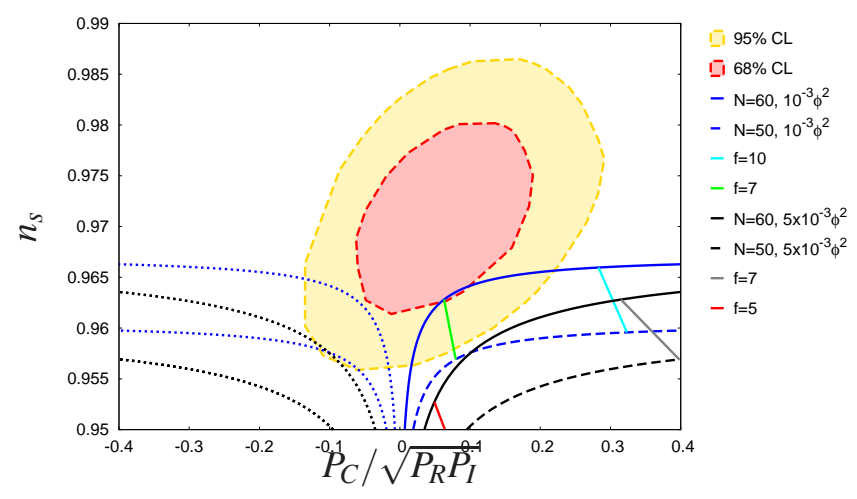

Figure 2: $P_{C} / \sqrt{P_{R} P_{I}}$ and the adiabatic spectral index $n_{s}$ for the natural inflation model $(68 \%$ and $95 \% \mathrm{CL}$ contours are from Planck). $P_{C} / \sqrt{P_{R} P_{I}}=c \times \phi_{0}^{2}$ for $c=10^{-3}, 5 \times 10^{-3}$ are shown for varying $N$ and $f$ (the labels are in units of the reduced Planck mass). The anti-correlation cases (for $c=-10^{-3},-5 \times 10^{-3}$ ) are also shown with the dotted curves.

The cross-correlation parameter $\beta_{C}$ is constrained to be $-0.1 \lesssim \beta_{C} \lesssim 0.3$, or, in terms of the parameters in the potential, to be within

$$
-0.1 \lesssim 4.2\left(\frac{1}{g_{1} g_{2}}\right)\left(\frac{\Lambda_{2}^{4}}{2.2 \times 10^{-9}}\right) \cos \left(\psi_{0}+\theta_{0}\right) \frac{\phi_{0}^{2}}{96 \pi^{2}} \lesssim 0.3 .
$$

We can also estimate the fraction of isocurvature perturbations

$$
\beta_{\text {iso }}=\frac{P_{I}}{P_{R}+P_{I}}=\frac{\frac{P_{I}}{P_{R}}}{1+\frac{P_{I}}{P_{R}}},
$$




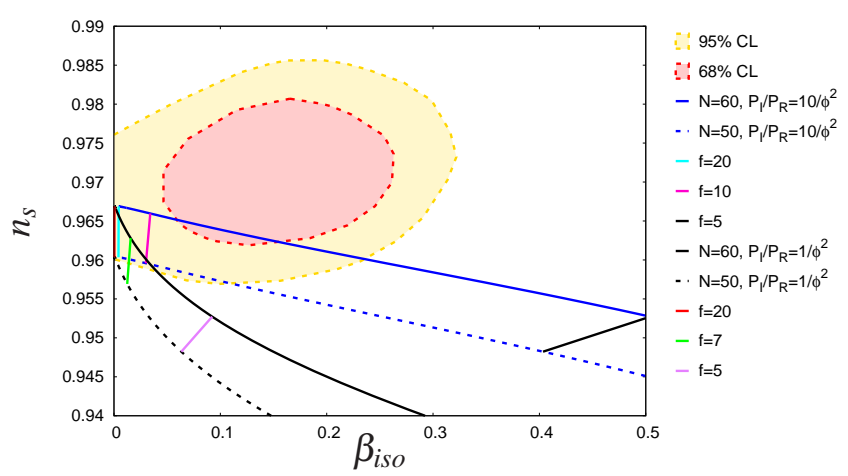

Figure 3: $\beta_{\text {iso }} \equiv P_{I} /\left(P_{R}+P_{I}\right)$ and $n_{s}$ for the natural inflation (68\% and 95\% CL contours are from Planck). $\left(\beta_{\text {iso }}, n_{s}\right)$ are shown for $P_{I} / P_{R}=c / \phi_{0}^{2}$ with $c=1,10$ for varying $f$ and $N$.

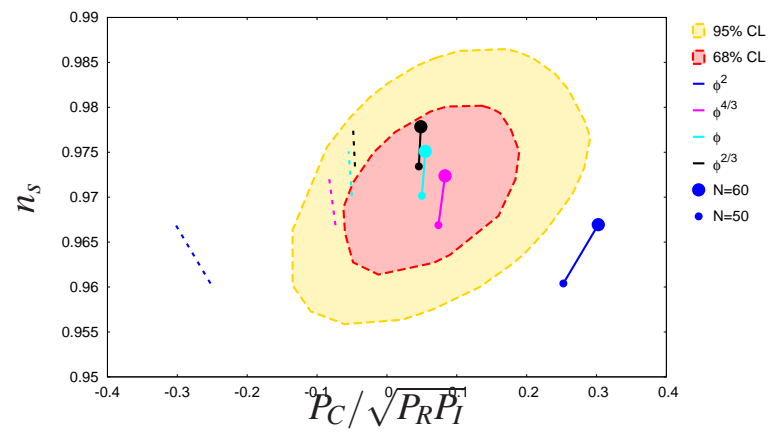

Figure 4: $P_{C} / \sqrt{P_{R} P_{I}}$ and $n_{s}$ for the axion monodromy inflation $\left(V_{\text {inf }}=\mu_{1}^{4-p} \phi^{p}\right)(68 \%$ and $95 \%$ CL contours are from Planck). $P_{C} / \sqrt{P_{R} P_{C}}=c \times \phi_{0}^{2} / p^{2}$ for $c=0.005$ are shown for varying the e-folding number $N$. The anti-correlation cases (for $c=-0.005$ ) are also shown with the dashed lines.

where the power spectrum of the adiabatic perturbation is fixed as in Eq. (3.7), whereas the power spectrum of the isocurvature perturbation is given by

$$
P_{I} \approx\left(\frac{\Omega_{a}}{\Omega_{m}}\right)^{2}\left(\frac{1}{2 \pi}\right)^{2}\left(\frac{2}{g_{1} \theta_{0}}\right)^{2} \frac{\Lambda_{1}^{4}}{6}\left(\frac{\phi_{0}^{2}}{f^{2}}\right)=\left(\frac{\Omega_{a}}{\Omega_{m}}\right)^{2}\left(\frac{1}{g_{1} \theta_{0}}\right)^{2} \frac{16 A_{S}}{\phi_{0}^{2}} .
$$

Then, the fraction of isocurvature perturbations

$$
\frac{P_{I}}{P_{R}} \approx 16\left(\frac{\Omega_{a}}{\Omega_{m}}\right)^{2}\left(\frac{1}{g_{1} \theta_{0}}\right)^{2} \phi_{0}^{-2}
$$

can give a sizable contribution to the cosmological observables as illustrated in Fig. 3 where the prefactor of $\phi_{0}^{-2}$ in Eq. (3.11) is set to 1 and 10 for a varying $f$. The Planck bounds the uncorrelated axion isocurvature mode to $\beta_{\text {iso }} \lesssim 0.038$, whereas the inclusion of isocurvature cross-correlation results in the constraint $0.034 \lesssim \beta_{\text {iso }} \lesssim 0.28$ at the 95\% confidence level [8].

It is straightforward to perform an analogous analysis for the more general cases such as the axion monodromy inflation scenarios $[9,10]$

$$
V=\mu_{1}^{4-p} \phi^{p}+\mu_{2}^{4} \cos \left(\frac{\phi}{g_{1}}+\frac{\chi}{g_{2}}\right),
$$


for which the natural inflation is a special case with $p=2$. For such axion monodromy inflation scenarios, we can obtain

$$
\begin{gathered}
\beta_{C}=\frac{P_{C}}{\sqrt{P_{R} P_{I}}}=2.1\left(\frac{1}{g_{1} g_{2}}\right)\left(\frac{\mu_{2}^{4}}{12 \pi^{2} A_{S}}\right) \cos \left(\psi_{0}+\theta_{0}\right)\left(\frac{\phi_{0}}{p}\right)^{2}, \\
\frac{P_{I}}{P_{R}} \approx\left(\frac{\Omega_{a}}{\Omega_{m}}\right)^{2}\left(\frac{2}{g_{1} \theta_{0}}\right)^{2}\left(\frac{p}{\phi_{0}}\right)^{2} .
\end{gathered}
$$

The cross-correlation parameter is shown in Fig. 4, and we can see that, for the axion monodromy inflation with $p=1,2 / 3$ including the sinusoidal corrections inducing the non-trivial crosscorrelations, there is a preference for the existence of cross-correlated isocurvature modes in the currently available CMB data.

We discussed in this presentation the forecasts for the CMB sensitivity on the scale dependent cross-correlated isocurvature perturbations which are generically expected in existence of the ubiquitous scalar fields in the early Universe. We, for concreteness, illustrated the phenomenology of the scale dependent cross-correlation power spectrum for the axion inflation scenarios. The cross correlation spectra can affect the preferable inflationary parameter regions to be consistent with the observables such as the CMB data, and they can give us the useful insight on the early universe dynamics where there presumably existed many light degrees of freedom inducing the non-trivial cross correlations among their fluctuations.

\section{Acknowledgments}

The author thanks Jinn-Ouk Gong, Kiyomoto Ichiki, Tatsuo Kobayashi, Takahiko Matsubara and Hajime Otsuka for the collaborations. This work was partly supported by Institute for Basic Science (IBS-R018-D1).

\section{References}

[1] K. Kadota, J. O. Gong, K. Ichiki and T. Matsubara, JCAP 1503, no. 03, 026 (2015) doi:10.1088/1475-7516/2015/03/026 [arXiv:1411.3974 [astro-ph.CO]].

[2] K. Kadota, T. Kobayashi and H. Otsuka, JCAP 1601, no. 01, 044 (2016) doi:10.1088/1475-7516/2016/01/044 [arXiv:1509.04523 [hep-ph]].

[3] A. Lewis and A. Challinor, Phys. Rept. 429, 1 (2006) [astro-ph/0601594].

[4] P. A. R. Ade et al. [Planck Collaboration], arXiv:1303.5082 [astro-ph.CO].

[5] P. A. R. Ade et al. [Planck Collaboration], Astron. Astrophys. (2014) [arXiv:1303.5076 [astro-ph.CO]].

[6] K. Freese, J. A. Frieman and A. V. Olinto, Phys. Rev. Lett. 65 (1990) 3233.

[7] S. Weinberg, Phys. Rev. D 72 (2005) 043514 [hep-th/0506236].

[8] P. A. R. Ade et al. [Planck Collaboration], arXiv:1502.02114 [astro-ph.CO].

[9] L. McAllister, E. Silverstein and A. Westphal, Phys. Rev. D 82 (2010) 046003 [arXiv:0808.0706 [hep-th]].

[10] E. Silverstein and A. Westphal, Phys. Rev. D 78 (2008) 106003 [arXiv:0803.3085 [hep-th]]. 